\title{
História Familial e Câncer Colorretal em Idade Precoce: Deve-se Indicar Colectomia Estendida?
}

\author{
Family History of Colorectal Cancer at Early Age: Should Extended \\ Colectomy be Indicated?
}

\author{
RENATO DE ARAÚJO BONARDI ${ }^{1}$, MARIACRISTINA SARTOR ${ }^{2}$, ANTONIO BALDIN JR. ${ }^{3}$, GUILHERME MATTIOLI \\ $\mathrm{NICOLLELLI}^{4}$, JOÃO RICARDO DUDA ${ }^{4}$, MÁRCIA OLANDOSKI $^{5}$
}

\begin{abstract}
${ }^{1}$ Prof. Adjunto Doutor do Depto. de Cirurgia e Chefe dos Serviços de Cirurgia Geral e Coloproctologia da UFPR; ${ }^{2}$ Doutora em Cirurgia do Aparelho Digestivo pela USP. Médica da Unidade de Coloproctologia do HC UFPR; ${ }^{3}$ Mestre em Microbiologia, Parasitologia e Patologia pela UFPR. Título de Especialista em Coloproctologia. Médico da Unidade de Coloproctologia do HC UFPR $;{ }^{4}$ Médicos Residentes da Unidade de Coloproctologia do Hospital de Clínicas da UFPR; ${ }^{5}$ Professora de Bioestatística da Pontifícia Universidade Católica do Paraná - PUCPR - Brasil.
\end{abstract}

BONARDI RA; SARTOR MC; BALDIN JR A; NICOLLELLI GM; DUDA JR; OLANDOSKI M. História Familial e Câncer Colorretal em Idade Precoce:Deve-se Indicar Colectomia Estendida? Rev bras Coloproct, 2006;26(3): 244-248.

RESUMO: Objetivo - avaliar a história familial e as características dos tumores em dois grupos distintos por sua faixa etária, bem como questionar quanto à necessidade de colectomia estendida, baseando-se nestas características. Método - Em estudo retrospectivo analisaram-se 106 pacientes por meio do prontuário e de contato telefônico com o próprio doente e/ou familiares, operados no HC-UFPR. Foram divididos em 2 grupos: Grupo A (n=51) com 55 anos ou menos, e Grupo B (n=55) com mais de 55 anos. Avaliou-se o número de doentes com parentes de $\mathbf{1}^{\circ}$. grau com câncer colorretal e em outros sítios; o número de parentes com CCR; o sítio do tumor; o estadiamento TNM; os óbitos no período e a presença de neoplasias associadas. Resultados - No grupo A, 16 (31,4\%) pacientes tinham parentes com câncer colorretal. No grupo B, sete $(\mathbf{1 2 , 7 \%})(\mathrm{p}=\mathbf{0 , 0 3 2})$. No grupo A houve 16 (31,4\%) pacientes de familiares com outro tipo de neoplasia. No grupo $\mathrm{B}, 19(34,5 \%)$ doentes $(\mathrm{p}=\mathbf{0 , 8 3 7})$. Ao incluírem-se apenas as neoplasias associadas à Síndrome do HNPCC, como endométrio e estômago, houve $5(9,8 \%)$ pacientes no grupo A e $3(5,9 \%)$ no grupo $B(p=0,477)$. No grupo $A, 3(5,8 \%)$ doentes apresentaram neoplasias associadas ao CCR, sendo elas estômago, rim e bexiga. No grupo B, 3 (5,4\%) doentes, mas os tumores eram de mama e próstata (p=0,624). Predominou no grupo A pacientes com estádio III (41,2\%) e no grupo B, o estádio II (51,9\%) (p=0,480). Houve 19,6\% de óbitos no grupo Ae $32,7 \%$ no Grupo B $(p=0,185)$. Conclusão - Pacientes com idade $=55$ anos possuem história familial mais representativa para o CCR que pacientes mais idosos, podendo fazer parte da síndrome do HNPCC. Possivelmente se beneficiem de colectomia estendida.

Descritores: história familial - idade - câncer colorretal - colectomia.

\section{INTRODUÇÃO}

O câncer colorretal (CCR) é a terceira causa mais comum de mortes por câncer ${ }^{1,2}$. A hereditariedade é responsável por 3 a $5 \%$ dos casos de câncer colo-retal.2,3. A Síndrome de Lynch, também conhecida como HNPCC (câncer colorretal hereditário não polipóide), representa modelo importante deste meca- nismo. Nestes casos, observa-se o surgimento da neoplasia em idades mais precoces, em média aos 45 anos, e predileção pelo cólon direito . 2,4,5,6.

A história familial e a faixa etária abaixo de 50 anos podem fornecer dados importantes para a presença da síndrome de Lynch. No entanto, o número reduzido e de registro adequado das famílias atuais, pode dificultar o diagnóstico, baseando-se apenas na história

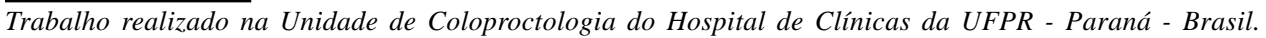

Recebido em 14/06/2006

Aceito para publicação em 08/08/2006 
familial (critérios de Amsterdam II) ${ }^{2}$. A avaliação genética pode ser necessária. Porém, devido aos custos, não está disponível para a maioria da população. Instabilidade de microssatélite está presente em 90 a $95 \%$ dos pacientes com a síndrome de Lynch e, como primeiro estudo, podem direcionar a avaliação para a pesquisa de alterações nos genes de reparo do DNA relacionados à Síndrome de Lynch, como o MLH1, MSH2 e MSH6 ${ }^{2,7,8}$. Pacientes em que o diagnóstico de câncer colorretal é feito antes dos 55 anos devem sempre ser investigados para uma história familial bem detalhada, pois podem ser portadores das Síndromes do HNPCC ou da Polipose Adenomatosa Familial. Outros cânceres devem ser investigados, pois fazem parte da Síndrome do HNPCC, como o de endométrio, ovário, gástrico, ou do trato urinário .,2,9,10.

A maioria dos autores recomenda que os exames de rastreamento para os familiares se iniciem aos 40 anos ou 10 anos antes da idade do familiar afetado. $7,10,11,12$ No Brasil existem deficiências para realizar exames de rastreamento para o câncer colorretal, seja por fatores financeiros ou desinformação dos médicos e da população ${ }^{13}$. Baseado nesta afirmação, este estudo objetiva avaliar o perfil dos pacientes com câncer colorretal dentro de dois grupos etários distintos, enfatizando a história familial e a síndrome do HNPCC como análises principais. Objetivou-se também realizar o seguinte questionamento: estaria indicada colectomia estendida em pacientes jovens e com história familial positiva?

\section{MÉTODO}

Realizou-se estudo longitudinal, retrospectivo, por meio de revisão de prontuários e de contato direto com pacientes e/ou respectivos familiares que foram submetidos à ressecção de câncer colorretal por adenocarcinoma, de janeiro de 2001 a março de 2006, na Unidade de Coloproctologia do Hospital de Clínicas da Universidade Federal do Paraná. (HC UFPR)

O estudo foi aprovado pelo Comitê de Ética e Pesquisa em Seres Humanos do HC UFPR.

Foram avaliados 106 pacientes operados por adenocarcinoma colorretal. Todas as ressecções foram realizadas através de laparotomia convencional. Indivíduos com Polipose Adenomatosa Familial foram excluídos do estudo.

Os pacientes foram divididos em 2 grupos: Grupo A ( $\mathrm{n}=51)$ de pacientes com 55 anos ou menos, e
Grupo B ( $\mathrm{n}=55)$, com idade maior que 55 anos, num total de 106 pacientes. Em cada grupo foi avaliado o número de doentes com parentes de $1^{\circ}$. grau (pais, irmãos e filhos) com câncer colorretal e em outros sítios. Também foram analisados o número de parentes com CCR; o sítio do tumor; o estadiamento TNM; o número de óbitos no período e a presença de neoplasias associadas. Estes dados relacionavam-se ao momento da operação.

No grupo A, a média de idade dos pacientes foi de $45 \pm 7$ anos. No grupo B, a média de idade foi de $70 \pm 7$ anos.

Os dados foram submetidos à análise estatística. Para a comparação entre os grupos quanto ao número de pacientes com parentes portadores de câncer de intestino, utilizou-se o teste exato de Fischer bilateral. O mesmo teste foi utilizado para análise dos óbitos no período. O teste do Qui-Quadrado foi o escolhido para análise do sítio tumoral e do estadiamento. $\mathrm{O}$ valor $\mathrm{p}=0,05$ foi considerado estatisticamente significativo.

\section{RESULTADOS}

No grupo A, $16(31,4 \%)$ pacientes tinham parentes com câncer colorretal. No grupo B, sete (12,7\%). Esta diferença foi estatisticamente significativa (tabela1).

Outro dado observado foi o fato de que 5 dos 16 pacientes com CCR do Grupo A possuíam dois parentes de $1^{\circ}$. grau com CCR. Foi estatisticamente significativa a diferença entre os grupos em relação a ter dois parentes de $1^{\circ}$. grau com CCR (tabela 2 ).

Ao avaliar-se o número de pacientes com familiares com outros tipos de neoplasia, observou-se que no grupo A houve 16 (31,4\%) pacientes com história positiva para tal. Já no grupo B, foram 19 (34\%) doentes $(\mathrm{p}=8368)$. No entanto, ao incluirem-se apenas as

Tabela 1 - Número de doentes com parentes com CCR.

\begin{tabular}{lcc}
\hline $\begin{array}{l}\text { Parentes } \\
\text { com CCR }\end{array}$ & $\begin{array}{c}\text { Grupo A } \\
\mathbf{n = 5 1}\end{array}$ & $\begin{array}{c}\text { Grupo B } \\
\mathbf{n = 5 5}\end{array}$ \\
\hline Sim & $16(31,4 \%)$ & $7(12,7 \%)$ \\
Não & $35(68,6 \%)$ & $48(87,3 \%)$ \\
\hline
\end{tabular}

Valor de $\mathbf{p = 0 , 0 3 2}$ (CCR: câncer colorretal). 
neoplasias associadas à Síndrome do HNPCC, como endométrio e estômago, houve $5(9,8 \%)$ pacientes no grupo A. No grupo B, apenas $3(5,4 \%)$ doentes. Este dado não mostrou significância estatística $(\mathrm{p}=0,4772)$.

No grupo A, houve $3(5,8 \%)$ doentes com neoplasias associadas ao CCR, sendo elas estômago, rim e bexiga. Por outro lado, no grupo com idade maior que 55 anos, houve também $3(5,4 \%)$ doentes com estas características, mas os tumores eram de mama e próstata $(\mathrm{p}=1)$.

Quanto ao sítio do tumor, comparando-se os grupos, notou-se a seguinte distribuição. No grupo A, $35 \%$ localizavam-se no cólon direito, $15 \%$ no cólon transverso, $12 \%$ no cólon esquerdo, $20 \%$ no cólon sigmóide e $29 \%$ no reto. No grupo B $34 \%$ situavam-se no cólon direito, $5 \%$ no cólon transverso, $11 \%$ no cólon esquerdo, $34 \%$ no sigmóide e $20 \%$ no reto (tabela 3 ). A soma dos percentuais é maior do que $100 \%$ devido à presença de tumores sincrônicos, que se encontravam em 7,8\% (4) dos pacientes do grupo A e em 7,2\% (4) pacientes do grupo B.

A avaliação comparativa do estadiamento TNM também não mostrou diferença estatisticamente significativa. Os dados são estratificados na tabela 4:

O número de óbitos no período foi proporcionalmente maior no grupo $\mathrm{B}$, como mostram os valores na tabela 5:

\section{DISCUSSÃO}

O câncer colo-retal corresponde a uma parcela importante de mortes e doenças relacionadas ao câncer no mundo. A população geral tem sido orientada a iniciar exames de rastreamento aos 50 anos. Indivíduos com síndromes familiares como o HNPCC e a Polipose Adenomatosa Familial devem iniciar rastreamento com colonoscopia mais brevemente. No entanto, tais síndromes respondem por apenas $5 \%$ dos CCR. A história familial deve sempre ser obtida com atenção para detectar possíveis pacientes com estas síndromes e submetê-los a exames de rastreamento com colonoscopia ${ }^{1-12}$.

Este estudo traça um perfil de uma população de pacientes operados por câncer colorretal baseando-se principalmente na história familial e na idade dos pacientes.

Por meio deste estudo foi possível demonstrar que grupos mais jovens de pacientes com CCR possuem maior número de parentes com câncer colorretal e
Tabela 2 - número de parentes com CCR.

\begin{tabular}{|c|c|c|}
\hline $\begin{array}{l}\text { Dois parentes } \\
\text { com CCR }\end{array}$ & $\begin{array}{c}\text { Grupo A } \\
\mathrm{n}=51\end{array}$ & $\begin{array}{c}\text { Grupo B } \\
\mathrm{n}=55\end{array}$ \\
\hline Sim & $5 \quad(9,8 \%)$ & $0 \quad(0 \%)$ \\
\hline Não & $46(90,2 \%)$ & $55(100 \%)$ \\
\hline
\end{tabular}

Valor de $p=0,0232$.

Tabela 3 - distribuição quanto ao sítio tumoral.

\begin{tabular}{lrr}
\hline Colon & Grupo A & Grupo B \\
\hline Direito & $18(35 \%)$ & $19(34 \%)$ \\
Transverso & $8(15 \%)$ & $3(5 \%)$ \\
Esquerdo & $6(12 \%)$ & $6(11 \%)$ \\
Sigmóide & $10(20 \%)$ & $19(34 \%)$ \\
Reto & $15(29 \%)$ & $11(20 \%)$ \\
\hline
\end{tabular}

Tabela 4 - estadio TNM: comparação entre os grupos.

\begin{tabular}{lcc}
\hline Estádio & Grupo A & Grupo B \\
\hline I & $7(14 \%)$ & $7(13 \%)$ \\
II & $19(37 \%)$ & $28(51 \%)$ \\
III & $21(41 \%)$ & $18(33 \%)$ \\
IV & $4(8 \%)$ & $2(3 \%)$ \\
\hline
\end{tabular}

$\boldsymbol{P}=0,48 * *$ agrupando-se estádios III e IV.

Tabela 5 - Proporção de óbitos no período em estudo.

\begin{tabular}{lll}
\hline Óbitos & Grupo A & Grupo B \\
\hline Sim & $10(20 \%)$ & $18(33 \%)$ \\
Não & $41(80 \%)$ & $37(67 \%)$ \\
\hline
\end{tabular}

$p=0,185$.

maior probabilidade destes pertencerem à síndrome familiar do HNPCC. Estudos como os de Eissen et al., Kievit et al., Hampel et al., Lynch et al e MuellerKoch et al. relatam estas características. No tocante à média de idade, nossa população mais jovem apresentou a média de 45 anos para o diagnóstico do câncer, de acordo com a maioria dos estudos. 2,7, 8,10, 14 . 
Quanto à distribuição do sítio tumoral observou-se uma discreta migração dos tumores para sítios mais proximais no grupo mais jovem, em especial para os tumores do cólon transverso. No entanto, a maioria dos estudos ressalta a predominância dos tumores do cólon direito nos pacientes com a síndrome do HNPCC, chegando a valores de até $70 \%{ }^{14,15}$.

Em referência ao estadiamento TNM, oestudomostrou que os pacientes do grupo A tiveram seu diagnóstico em fases mais avançadas. Este dado, porém, não foi significativo. Apesar disso, a mortalidade foi menor no grupo mais jovem, o que vem corroborar com a literatura - a qual cita que, apesar dos indicadores de mau prognóstico, o resultado é relativamente bom com o tratamento cirúrgico e complementar ${ }^{16,17}$.

Não houve significância estatística quanto às neoplasias associadas. Porém, no grupo de pacientes mais jovens, estas foram de estômago, rim e bexiga pertencentes à síndrome de Lynch; enquanto que nos mais idosos foram de mama e próstata ${ }^{9}$.

Este estudo comprova que se deve dar atenção ao histórico familiar e à idade do doente, uma vez que o acesso aos exames de estudo genético, como de instabilidade microssatélite e imunohistoquímica para avaliação de deleções de genes de reparo do DNA, como o MLH1, MSH2 e MSH6, é difícil. Além disso, estima-se que $15,8 \%$ dos pacientes com história fami- liar de CCR são parte do HNPCC. ${ }^{18 .}$ Assim, é necessário orientar os pacientes e suas famílias a participarem de programas de prevenção desde idades mais precoces e de maneira mais rígida.

Em casos de pacientes jovens e com história familial para CCR ou outros tumores pertencentes à síndrome do HNPCC, deve-se atentar para a realização de ressecções colônicas estendidas, seja em cirurgias eletivas, ou principalmente quando admitidos na emergência e sem estudo do cólon. Isso porque pacientes com HNPCC têm $30 \%$ de chance de desenvolver um segundo CCR em 10 anos e 50\% em 15 anos ${ }^{19,20 .}$ Quando os estudos genéticos confirmam a síndrome, a maioria dos autores preconiza colectomia subtotal com anastomose ileoanal ${ }^{21,22}$. Dessa forma, pode-se prevenir a negligência de não tratar possíveis tumores sincrônicos ou metacrônicos, afetando significativamente o prognóstico.

\section{CONCLUSÃO}

Pacientes com idade menor ou igual a 55 anos possuem história familial mais representativa para $\mathrm{o}$ CCR que pacientes mais idosos, podendo fazer parte da síndrome do HNPCC. Possivelmente se beneficiem de colectomia estendida.

\footnotetext{
ABSTRACT: Aim - To evaluate the family history and the characteristics of the tumors in two different groups of patients, divided by age, questioning the necessity of extensive colectomy for the adequate treatment. Method - One hundred and six patients were analyzed retrospectively by hospital chart review or phone contact either with the patient or a next of keen; all were operated at the University Hospital of the "Universidade Federal do Paraná" (Brazil).These patients were divided in Group A ( $(n=51)$ below 55 years old, and Group B $(n=55)$ with age above 55 years. All patients were analyzed for first degree relatives with history of colon and rectal cancer and cancer history in other organs; number of relatives with colon and rectal cancer; the location of the tumors in the colon; the TNM staging; the occurrence of other malignant neoplasias for each patient; and the death rate for the period of the study. Results - On group A, $16(31.4 \%)$ patients had relatives with colorectal cancer; on group $B, 7(12.7 \%)(p=0,032)$. On group $A, 16(31.4 \%)$ patients had relatives with other types of malignant neoplasias, and on group $B, 19(34.5 \%)(p=0.837)$. When it was considered only cancers related to the HNPCC syndrome, as endometrium and stomach, $5(9.8 \%)$ patients were found on group $A$ and $3(5.9 \%)$ on group $B(p=0.477)$. On group A, $3(5.8 \%)$ patients had tumors related to colon and rectal cancers, as stomach, kidney and bladder. On group B, $3(5.4 \%)$ patients, but these tumors were in the breast and prostate $(p=\mathbf{0 . 6 2 4})$. On group $A$, there were more patients with stage III cancers $(41.2 \%)$ and on group $B$, stage II cancers were more prevalent $(51.9 \%)(p=0.480)$. The death rate was $19.6 \%$ on group A and 32.7\% on group B (p=0.185). Conclusion - Patients under 55 years of age have an increased family history of colon and rectal cancer when compared to the ones above the age of 55; those could be related to the HNPCC syndrome. These patients can benefit from an extended colectomy.
}

Key words: family history; age; colorectal cancer; colectomy. 


\section{REFERÊNCIAS}

1. Jemal A, Murray T, Ward E. Cancer statistics. CA Cancer J Clin. 2005; 55:10-30.

2. Lynch HT, Lynch PM. Molecular screenimg for the Lynch syndrome better than family history? N Engl J Med 2005; 352(18): 1920-2.

3. Lynch HT, Dela Chapelle A. Genetic susceptibility to nonpoliposis colorectal cancer. J Med Genet 1999; 36:801-18.

4. Lynch HT, De La Chapelle A. Hereditary Colorectal cancer. N Engl J Med. 2003; 348:919-32.

5. Jass JR, Stewart SM, Stewart J, Lane MR. Hereditary nonpolyposis colorectal cancer - morphologies, genes and mutations. Mutat Res. 1994; 310:125-33.

6. Jass JR, Smyrk TC, Stewart SM. Pathology os hereditary non-polyposis colorectal cancer. Anticancer Res. 1994; 14:1631-4.

7. Kievit W, De Bruin JHFM, Adang EMM. Cost effectiveness of a new strategy to identify HNPCC patients. Gut 2005; 54:97-102.

8. Mueller-Koch Y, Vogelsang H, Kopp R. Hereditary nonpolyposis colorectal cancer: clinical and molecular evidence for a new entity of hereditary colorectal cancer. Gut 2005; 54:1733-40.

9. Douglas JA, Gruber SB, Meister KA, Lynch HT. History and molecular genetics of Lynch syndrome in Family G. JAMA 2005; 294:2195-202.

10. Eisen GM, Weiberg DS. Narrative review: screening for colorectal cancer in patients with a first-degree relative with colonic neoplasia. Ann Intern Med. 2005; 143:190-8.

11. Winawer S, Fletcher R, Rex D. Colorectal cancer screening and surveillance: clinical guidelines and rationale - update based on new evidence. Gastroenterology 2003; 1224:544-60.

12. Smith RA, Cokkinides V, Eyre HJ. American Cancer Society guidelines for early detection of cancer. CA Cancer J Clin. 2004; 54: 41-52.

13. Oliveira Ferreira F, Napoli Ferreira CC, Rossi BM, Toshihiko Nakagawa W, Aguilar S, Monteiro Santos EM, et al. Frequency of extra-colonic tumorsin hereditary nonpolyposis colorectal cancer (HNPCC) and familial colorectal cancer (FCC) Brazilian families: An analysis by a Brazilian Hereditary Colorectal Cancer Institutional Registry. Fam Cancer 2004; 3(1):41-7.

14. Patchett SE, Alstead EM, Saunders BP. Regional proliferative patterns in the colon of patients at risk for hereditary nonpolyposis colorectal cancer. Dis Colon Rectum 1997; 40:168-71.

15. Vasen, HF, Mecklin, JP, Khan, PM, Lynch, H T. The International Collaborative Group on Hereditary NonPolyposis Colorectal Cancer (ICG-HNPCC). Dis. Colon Rectum 1991;34: 424-5.

16. Giardiello, FM, Brensinger, JD, Petersen, GM. AGA technical review on hereditary colorectal cancer and genetic testing. Gastroenterology. 2001;121: 198-213.

17. Lynch, H. T. Hereditary nonpolyposis colorectal cancer (HNPCC). Cytogenet. Cell Genet. 1999; 86:130-5.

18. Gaçkia P, Atkin WS, Whitelaw S. Variables associated with the risk of colorectal adenomas in asymptomatic patients with a family history of colorectal cancer. Gut 1995; 36: 385-90.

19. Lynch HT, Harris RE, Lynch PE. Role of heredity in multiple primary cancer. Cancer 1977;40:1849-54.

20. Mecklin JP, Jarvinen HJ. Clinical features of colorectal carcinoma in cancer family syndrome. Dis Colon Rectum 1986;29:160-4.

21. Marra G, Boland CR. Hereditary nonpolyposis colorectal cancer: the syndrome, the genes, and historical perspectives. $\mathrm{J}$ Natl Cancer Inst 1995, 87:1114-25.

22. Vasen HF, Nagengast FM, Khan PM. Interval cancers in hereditary non-polyposis colorectal cancer (Lynch syndrome). Lancet 1995, 345:1183- 4

\section{Endereço para correspondência:} RENATO DE ARAÚJO BONARDI

Rua Olavo Bilac, 680

80.440-040 - Curitiba (PR)

Fone: (41) 3244-8963 - (41) 3232-1262

FAX: (41) 32438827

E-mail: rbonardi@onda.com.br 